\title{
Complicaciones en el posoperatorio de la angioplastia coronaria transluminal percutánea: una visión a los cuidados de enfermería
}

\author{
Postoperative complications of percutaneous transluminal coronary angioplasty: a view to nursing \\ care
}

Complicações pós-operatórias da angioplastia coronária transluminal percutânea: uma visão ao cuidado de enfermagem

Recibido: 03/09/2021 | Revisado: 11/09/2021 | Acepto: 14/09/2021 | Publicado: 17/09/2021

\author{
Judith Victoria Castillo Mejia \\ ORCID: https://orcid.org/0000-0001-5257-6878 \\ Faculdade Unyleya, Brasil \\ E-mail: Iker07castillo@live.com \\ Jonas Magno dos Santos Cesário \\ ORCID: https://orcid.org/0000-0003-1785-3555 \\ Faculdade Unyleya, Brasil \\ E-mail: prof.jonasmagno@unyleya.edu.br \\ Victor Hugo de Paula Flauzino \\ ORCID: https://orcid.org/0000-0001-5156-0030 \\ Faculdade Unyleya, Brasil \\ E-mail: prof.victorflauzino@unyleya.edu.br
}

\begin{abstract}
Resumen
Objetivos: Describir las principales complicaciones en el posoperatorio de la angioplastia coronaria transluminal percutánea e identificar los respectivos cuidados de enfermería. Metodología: revisión bibliográfica y estudio descriptivo, exploratorio con enfoque cualitativo. Desarrollado a partir de consultas en páginas oficiales como ser: Ministerio de Salud, Asociación Brasileña de Cardiología, Agencia Nacional de Vigilancia Sanitaria (ANVISA) y Consejo Regional de Salud (COREN). También se consultaron las bases de datos: CUIDEN, CINAHL, LILACS, SciELO y BDENF. Resultados: Aunque la angioplastia se considera un procedimiento seguro, existe la posibilidad de complicaciones en el postoperatorio. Las complicaciones vasculares periféricas aparecen en las primeras 24 horas posteriores al procedimiento y son las que causan mayor preocupación. Por lo tanto, es fundamental la actualización constante por parte del enfermero, para identificar precozmente estas complicaciones, con el objetivo de garantizar una atención segura y reducir los riesgos para el paciente.
\end{abstract}

Palabras clave: Cuidados de enfermeria; Angioplastia; Intervención coronaria percutánea; Infarto del miocardio.

\begin{abstract}
Objectives: To describe the main postoperative complications of percutaneous transluminal coronary angioplasty and to identify the respective nursing care. Methodology: bibliographic review and descriptive, exploratory study with a qualitative approach. Developed from consultations on official pages such as: Ministry of Health, Brazilian Association of Cardiology, National Agency for Health Surveillance (ANVISA) and Regional Health Council (COREN). The following databases were also consulted: CUIDEN, CINAHL, LILACS, SciELO and BDENF. Results: Although angioplasty is considered a safe procedure, there is the possibility of complications in the postoperative period. Peripheral vascular complications appear in the first 24 hours after the procedure and are theones that cause the greatest concern. Therefore, constant updating by the nurse is essential to identify these complications early, to guarantee safe care and reduce risks for the patient.
\end{abstract}

Keywords: Nursing care; Angioplasty; Percutaneous coronary intervention; Myocardial infarction.

\section{Resumo}

Objetivos: Descrever as principais complicações pós-operatórias da angioplastia coronária transluminal percutânea e identificar os respectivos cuidados de enfermagem. Metodologia: revisão bibliográfica e estudo descritivo, exploratório com abordagem qualitativa. Desenvolvido a partir de consultas em páginas oficiais como: Ministério da Saúde, Associação Brasileira de Cardiologia, Agência Nacional de Vigilância Sanitária (ANVISA) e Conselho Regional de Saúde (COREN). Também foram consultadas as seguintes bases de dados: CUIDEN, CINAHL, LILACS, SciELO e BDENF. Resultados: Embora a angioplastia seja considerada um procedimento seguro, existe a possibilidade de complicações no pós-operatório. As complicações vasculares periféricas aparecem nas primeiras 24 horas após o 
procedimento e são as que mais preocupam. Portanto, a atualização constante do enfermeiro é imprescindível para identificar precocemente essas complicações, a fim de garantir uma assistência segura e reduzir os riscos ao paciente.

Palavras-chave: Cuidados de enfermagem; Angioplastia; Intervenção coronária percutânea; Infarto do miocárdio.

\section{Introducción}

En 2016, las patologías cardiovasculares fueron responsables a nivel mundial por más de 16,7 millones de muertes, aproximadamente el $30 \%$ del total de muertes en todo el mundo. En Brasil, el 29,4\% de todas las muertes registradas fueron causadas por enfermedades cardiovasculares. Esto significa que más de 308 mil personas murieron, principalmente por ataque cardíaco y accidente cerebrovascular (ACV). La alta incidencia de este problema convierte a Brasil en uno de los diez países con mayor tasa de muertes por enfermedades cardiovasculares del mundo (Hilário et al., 2017; Mansur \& Favarato, 2016; Régis, Rosa \& Lunelli, 2017).

En este sentido, la angioplastia coronaria transluminal percutánea (ACTP) se presenta como un procedimiento quirúrgico invasivo que promete la revascularización miocárdica. Este procedimiento se utiliza para aumentar o liberar el flujo sanguíneo al corazón, a través de un acceso vascular (femoral, radial, braquial u otra arteria), utilizando un catéter con balón. Este catéter se guía a la ubicación bloqueada y libera un tubo de metal llamado Stents, el cual se coloca en la pared del vaso para desbloquear y liberar el flujo sanguíneo al tejido cardíaco (Lambert et al., 2017; Soares et al., 2014).

A pesar del desarrollo de la tecnología, en la angioplastia como en cualquier intervención quirúrgica aún existe riesgo de complicaciones, como la formación de trombos, hemorragias, rupturas de las arterias coronarias, entre otras. Ante estas complicaciones, son necesarias algunas intervenciones urgentes para reducir los riesgos para el paciente. Para ello, el profesional de enfermería debe estar capacitado y entrenado para prevenir posibles lesiones (Abizaid A, 2013: Santos et al., 2018).

En este contexto, el conocimiento sobre angioplastia, así como la identificación precoz de signos y síntomas de complicaciones en el postoperatorio, puede ayudar al enfermeiro/a en la elaboración de intervenciones, en función de las necesidades individuales de cada paciente post-angioplastia. De esta forma, es posible direccionar las acciones de enfermería de forma rápida, competente y humanizada.

Actualmente, en los grandes hospitales existen sectores que tratan las enfermedades cardíacas. Estos sectores se denominan unidades hemodinámicas y es donde se realizan algunos procedimientos cardíacos, como el cateterismo y la angioplastia transluminal percutánea (Régis, Rosa \& Lunelli, 2017).

Los sectores de la hemodinámica son de alta complejidad y requieren que el enfermeiro/a tenga la capacidad de realizar cuidados de enfermería basados en conocimientos científico-técnicos, a través de la elaboración de la sistematización de enfermería y estableciendo una relación entre el equipo de trabajo, familiares y pacientes.

En esta línea de razonamiento, el equipo de enfermería debe responsabilizarse además del cuidado integral del cliente, de promover la salud, el bienestar, el descanso y la comodidad. El cuidado de enfermería está relacionado con las necesidades básicas del cliente, con el objetivo de brindar una atención humanizada y de calidad (Dotto et al., 2017; Ferrari et al., 2014; Mosleh, Nidal \& Mona, 2017; Silva et al., 2017).

Si bien existe un importante avance tecnológico en el campo de la enfermería cardiovascular, se observa que la tecnología no excluye la necesidad de cuidados básicos de enfermería al cliente, ya que muchas veces el perfil técnico del enfermero hace que este cuidado no sea valorado (Cuoto, Cesário, Flauzino \& Mejia, 2020; Ferrari et al., 2014; Silva et al., 2017).

Por tanto, se considera fundamental el conocimiento del enfermeiro/a sobre las complicaciones en el postoperatorio de la angioplastia, su gravedad y las conductas para cada caso, con el objetivo de brindar una atención de calidad. Basado en esto, esta investigación tuvo como objetivo describir las principales complicaciones en el postoperatorio de la angioplastia coronaria transluminal percutánea e identificar los respectivos cuidados de enfermería. 


\section{Metodología}

Se trata de una revisión bibliográfica de la literatura y un estudio descriptivo y exploratorio con enfoque cualitativo. Un enfoque cualitativo se ocupa de los aspectos de la realidad que no se pueden cuantificar, centrándose en comprender y explicar la dinámica de las relaciones sociales (Cesário, Flauzino \& Mejia, 2020). La siguiente figura describe el diagrama de flujo (PRISMA) de la primera etapa de la investigación.

Figura 1. Diagrama de flujo de la primera etapa de la investigación.

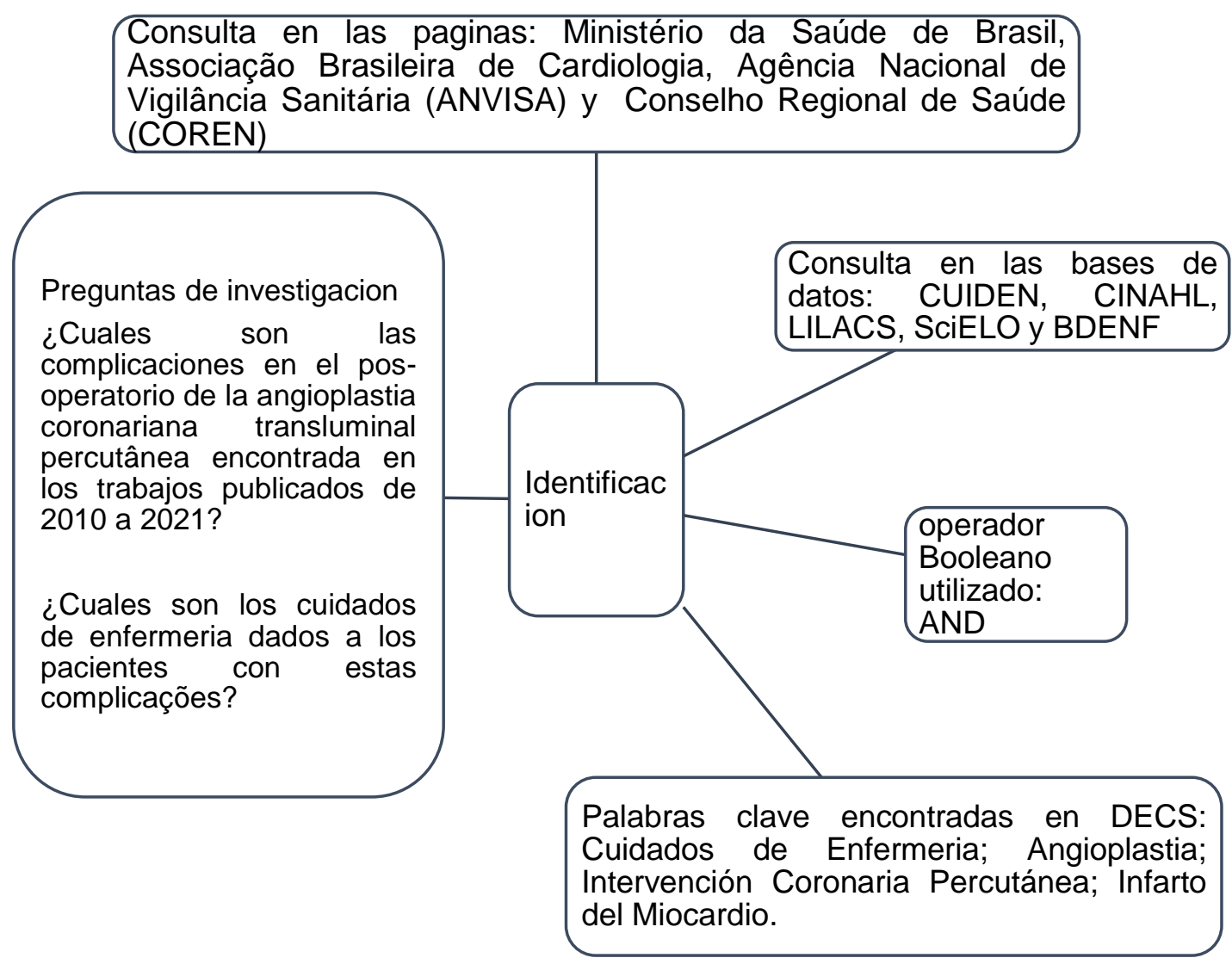

Fuente: Elaboración propia (2021).

La siguiente Figura 2, describe el diagrama de flujo (PRISMA) de las siguientes etapas de la investigación. 
Figura 2. Diagrama de flujo (PRISMA) de las siguientes etapas de la investigación.
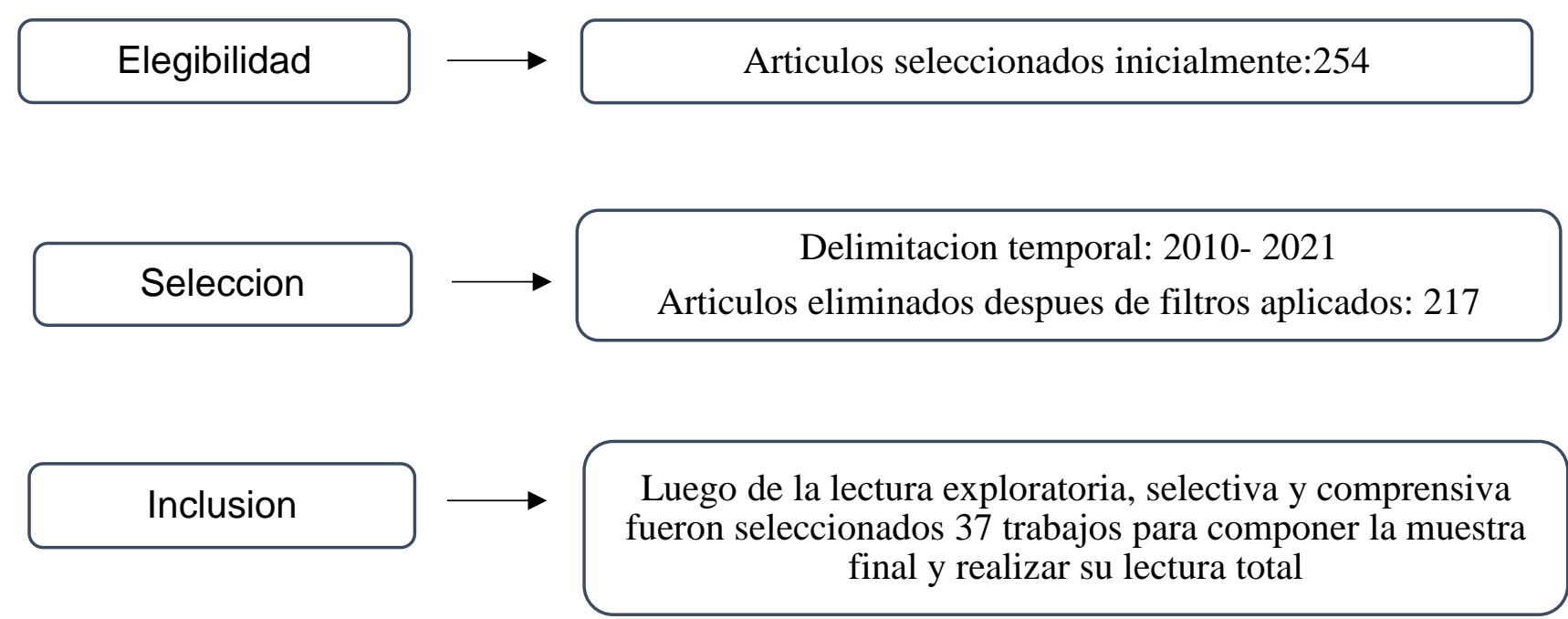

Fuente: Elaboración propia (2021).

\section{Resultados y Discusión}

Los resultados mostraron que las enfermedades cardiovasculares (ECV) son la principal causa de muerte en la población brasileña y son responsables del mayor número de ingresos hospitalarios, discapacidad y muerte. Se encontró que la mayoría de las personas de la tercera edad tienen al menos algún trastorno de la circulación de las arterias coronarias y que la mayoría de las muertes por enfermedades cardiovasculares se podrían prevenir si la población con cambios en el estilo de vida (Mansur \& Favarato, 2012; Szelkowski, Puri, Singh \& Massimiano, 2015).

En el caso de los factores de riesgo, estos se clasifican en factores de riesgo modificables y no modificables, siendo su control la mejor forma de prevenir complicaciones (Almeida, 2017; Kubrusly, 2010;).

Entre los principales factores de riesgo no modificables se encuentran: edad mayor de 55 años, antecedentes de ECV, sexo masculino y genética. Los factores de riesgo modificables son: dislipidemia, hipertensión arterial sistémica (HTA), obesidad, diabetes mellitus (DM), tabaquismo, inactividad física y estrés (Brunori et al., 2014; Graeff, Goldmeier \& Pellanda, 2012).

En este sentido, la identificación temprana de estos factores puede prevenir o reducir el número de muertes o morbilidades derivadas de esta enfermedad. Una vez confirmado el diagnóstico, el paciente puede realizar otras pruebas de imagen como el cateterismo y la angioplastia para poder investigar el tamaño de la lesión miocárdica. En los grandes hospitales, cuando el paciente ingresa con el cuadro clínico característico, es enviado a la unidad de hemodinámica, donde se realiza el procedimiento de cateterismo, aumentando la supervivencia de los pacientes. Sin embargo, lamentablemente esta posibilidad aún no es la realidad de todos los hospitales brasileños (Brunori et al., 2014; Silva, Pesaro, Franken \& Wajngarten, 2015).

Algunos de los procedimientos para el diagnóstico de esta patología son la Cinecoronariografía o Cateterismo Cardíaco. Este es un examen invasivo, que se puede realizar tanto de forma urgente como electiva. Tiene como objetivo confirmar la presencia de lesiones y obstrucciones de las arterias coronarias, para evaluar el funcionamiento de las válvulas y el músculo cardíaco, especialmente en situaciones de emergencia cuando se necesita confirmar la ubicación de la obstrucción que está dando lugar al Infarto Agudo de Miocardio (IAM) (Ponikowski et al., 2016).

El cateterismo cardiaco se realiza mediante una punción en la arteria radial o femoral, por parte del cardiólogo intervencionista. Este catéter es guiado al corazón mediante un equipo especial de rayos X. Durante el procedimiento, se inyecta 
contraste para que el médico pueda ver las arterias coronarias y dónde se encuentra la obstrucción. Después del procedimiento, dependiendo del resultado, se evalúa la necesidad de una angioplastia para el tratamiento (Régis, Rosa \& Lunelli, 2017; Silva, Pesaro, Franken \& Wajngarten, 2015).

Andreas Gruentzig en 1977 utilizó la angioplastia coronaria transluminal percutánea (ACTP), convirtiéndose en el método estándar de oro para aliviar la estenosis de los vasos y restaurar el flujo sanguíneo para disminuir los síntomas de la isquemia miocárdica. Antes, lo que se trataba solo con intervenciones quirúrgicas como la toracotomía, se comenzó a tratar con un catéter balón, representando un gran avance para la medicina y la salud (Piegas \& Haddad, 2010; Régis, Rosa \& Lunelli, 2017; Silva, Pesaro, Franken \& Wajngarten, 2015).

En Curitiba, Brasil, el 11 de agosto de 1979, el Dr. Constantino Constantini realizó con éxito la primera angioplastia coronaria (ACTP). De esta manera se inició la década de 1980, donde hubo una explosión en el uso de la ACTP, considerada la forma ideal de resolver el problema de las obstrucciones coronarias. Los años siguientes fueron ricos en la aparición de técnicas y dispositivos que se anunciaron como soluciones. En 1994, el Palmaz-Schatz stent fue aprobado por la Administración de Alimentos y Medicamentos (FDA), para su uso en Estados Unidos y su uso se difundió entre la profesión médica, entre los años 1994 y 1997 (Issa et al., 2015).

Aún sobre esto, se sabe que existen 2 tipos de stents: los stents farmacológicos y los no farmacológicos. Sin embargo, debido al alto costo de los stents liberadores de fármaco, hoy en Brasil, en la mayoría de los hospitales, solo se implantan stents no liberadores de fármaco, ya que el Sistema Único de Salud (SUS) solo reembolsa este modelo (Piegas \& Haddad, 2010).

\section{Complicaciones postoperatorias de la angioplastia coronaria transluminal percutánea (ACTP)}

Aunque la angioplastia se considera un procedimiento seguro, pueden ocurrir complicaciones. Dentro de las principales complicaciones vasculares periféricas en el postoperatorio de ACTP, se destacaron eventos hemorrágicos, formación de trombos o pseudoaneurismas (relacionados con el uso de catéter guía de mayor calibre, edad avanzada y uso de dosis más altas de heparina durante el período procedimiento), disección de arterias coronarias y oclusión aguda de arterias coronarias, siendo esta última la complicación más grave que puede presentar un paciente en el postoperatorio de ACTP durante las primeras 24 horas posteriores al procedimiento (Abizaid, 2013; Campos, 2011; Soares et al., 2011).

La angiografía coronaria se puede realizar a través de arterias femorales, radiales, braquiales, cubitales o axilares, sin embargo, la vía más utilizada son las arterias femorales y radiales. Cuando la ACTP se realiza por vía femoral la tasa de complicaciones puede ser mayor, esto se debe a que muchos pacientes, al ser sometidos a angioplastia, ya utilizan anticoagulantes y antiagregantes plaquetarios, aumentando así el riesgo de sangrado y consecuentemente el tiempo de hospitalización (Assunçâo \& Oliveira, 2012; Shore-Lesserson et al., 2018).

Al comparar la tasa de complicaciones, cuando la angioplastia se realiza por vía radial, estas son menores en comparación cuando se realiza por la vía femoral. A pesar de la tasa de complicaciones por vía femoral, esta vía se utiliza en aproximadamente el $90 \%$ de los procedimientos realizados, siendo las complicaciones por el uso de esta vía: hematomas, sangrado, pseudoaneurisma, trombosis e infección (Almeida, 2017; Barbosa et al., 2013; Ponikowski et al., 2016).

Otra complicación frecuente tras la angioplastia es la re-estenosis, por lo que se realizan grandes esfuerzos por conocer la fisiopatología de esta complicación. En este sentido, la re-estenosis coronaria post-angioplastia ocurre en alrededor del 30 al $40 \%$ de los casos y se considera cuando el grado de obstrucción del vaso, en la zona previamente dilatada, es mayor al 50\%, en un nuevo estudio angiográfico. Esto depende fundamentalmente de la proliferación fibromuscular que siempre sigue a la lesión que sufre la pared arterial en sus capas endotelial y muscular. Su comprensión fisiopatológica es uno de los desafíos más fascinantes de la medicina moderna y es necesaria una mejor comprensión de su patogenia para diseñar una estrategia adecuada para su control (Alves et al., 2013; Piegas \& Haddad, 2010). 
En este contexto, se diseñó el prototipo del catéter de aterectomía direccional con el objetivo inicial de reducir las tasas de re-estenosis. Este catéter, consiste en un sistema percutáneo que utiliza un balón con una ventana donde se deposita el material a retirarlo, mediante un sistema de corte realizado por una guillotina, que es accionado por un motor conectado externamente al catéter y que, al ser activado por el operador, realiza un corte lento con una rotación de $2000 \mathrm{rpm}$. Todo el sistema se mueve sobre una guía de 0,014 pulgadas. El material retirado del ateroma se deposita en la ventana, con previo inflado del balón, repitiendo la operación y rotando con reorientación del catéter, hasta conseguir un buen resultado angiográfico (Barbosa et al., 2013)

Por tanto, podemos observar que la re-estenosis es un problema común que aún no ha sido resuelto, lo que puede limitar la indicación del procedimiento. Ante estas complicaciones, se requieren algunas intervenciones urgentes con el fin de reducir los riesgos para el paciente, requiriendo la capacitación del profesional de enfermería para identificar precozmente los síntomas anormales, con el objetivo de disminuir la tasa de complicaciones en el postoperatorio, mediante acciones rápidas competentes y humanizadas por el equipo de enfermería (Régis, Rosa \& Lunelli, 2017).

\section{Atención de enfermería en el postoperatorio de la angioplastia coronaria transluminal percutánea (ACTP)}

Las Unidades de Hemodinámica se consideran sectores de alta complejidad que requieren de las habilidades y competencias del enfermero/a. El cuidado de enfermería al paciente en el post-operatorio de angioplastia juega un papel fundamental y crucial en su evolución, ya que inicialmente permite al paciente y familiares aclarar cualquier duda sobre el procedimiento y los cuidados necesarios en el postoperatorio para minimizar las complicaciones (Cheregatti \& Amorim, 2010; Costa et al., 2014; Costa, Cesário, Flauzino \& Mejia, 2021; Chaves, Brusamarello \& Huernermann, 2018; Pereira, Flauzino, Mejia \& Cesário, 2021; Silva, Cesário, Flauzino \& Mejia, 2020).

Todos los pacientes deben ser observados durante al menos 24 horas después del procedimiento y deben ser instruidos sobre la importancia del reposo absoluto, la caminata supervisada 12-24 horas después del procedimiento, instruyendo al paciente para que no flexione la extremidad inferior en la que se encontraba. Una vez realizado el procedimiento quirúrgico, el paciente no puede permanecer sentado y debe elevarse la cabeza a $45^{\circ}$. El equipo de enfermería debe medir la presión Arterial, controlar el pulso en el sitio de la punción, verificar el color y la temperatura de la extremidad, observar sangrado y hematomas. Cuando ocurren sangrado, se debe comprimir el sitio con suficiente presión hasta que el sangrado se detenga, luego realizar un vendaje compresivo y comunicarse con el equipo médico. Se debe instruir al equipo para que afloje el vendaje después de 6 horas del procedimiento, realizar un ECG post-procedimiento y retirar el apósito después de 24 horas (Assunçâo \& Oliveira, 2012; Agarwal, 2012; Costa, Cesário, Mejia \& Flauzino, 2021; Ferreira et al., 2016; Rios, Flauzino \& Cesário, 2021; Sousa, Flauzino \& Cesário, 2020).

Cabe destacar que, si bien el paciente debe descansar 12 horas después del procedimiento, nada le impide realizar el cambio de decúbito, aunque sea de forma limitada, con el fin de aliviar la presión sobre las prominencias óseas. Por lo tanto, corresponde al equipo de enfermería orientar al paciente y a la familia sobre este movimiento, con el objetivo de evitar lesiones derivadas de la incapacidad de moverse (Duarte, Stipp, Mesquita \& Silva, 2012; Farias, Vidal, Farias \& Jesus, 2013; Santos \& Cesário, 2019; Silva et al., 2017; Silva, Pesaro, Franken \& Wajngarten, 2015).

Vemos la importancia de orientar al paciente y familiar que, luego del alta hospitalaria, debe evitar cargar peso durante al menos 10 días y, además, higienizar el sitio de punción con agua y jabón durante el baño, luego mantener seco el lugar. Orientar cuánto evaluar en busca de signos de infección como hiperemia, presencia de secreciones, edema, hematoma extenso y fiebre y si presenta estos síntomas, acuda inmediatamente al servicio de salud (Cuoto, Cesário, Flauzino \& Mejia, 2020; Lima et al., 2021; Régis, Rosa \& Lunelli, 2017). 
Es necesario también, asesorar sobre hábitos para mejorar la calidad de vida y evitar alimentos que pueden elevar el colesterol. Hábitos como el tabaquismo aumentan el riesgo de re-estenosis tras la implantación de un stent, por lo que también debe evitarse. Enfatizar también sobre la importancia y necesidad de tomar todos los medicamentos recetados por el médico y regresar al médico de forma rutinaria (Costa, Cesário, Mejia \& Flauzino, 2021; Duarte, Stipp, Mesquita \& Silva, 2012; Régis, Rosa \& Lunelli, 2017; Ribeiro et al., 2021).

\section{Consideraciones Finales}

Los resultados mostraron que aún existen brechas de conocimiento sobre el tema. Además, fue posible describir las principales complicaciones que pueden presentarse en el postoperatorio de la angioplastia e identificar los respectivos cuidados de enfermería posteriores al procedimiento. Se espera que este trabajo estimule el estudio y producción de nuevos trabajos científicos, con el objetivo de dotar al equipo de enfermería con evidencia científica que contribuya a reducir las tasas de complicaciones en el postoperatorio de angioplastia en la unidad de hemodinámica.

Considerando la práctica basada en la evidencia, vemos la importancia de la actualización constante por parte del enfermero/a, para identificar de manera temprana estas complicaciones, con el objetivo de garantizar una atención segura y reducir los riesgos para el paciente. También es fundamental que las enfermeras/os participen en el plan terapéutico del paciente, prescribiendo cuidados de enfermería en el período pre, trans y postoperatorio de la ACTP, así como orientar al equipo multidisciplinario en la atención al paciente, con el fin de evitar futuras complicaciones.

\section{Referencias}

Abizaid, A. (2013). Manual de Cardiologia Intervencionista do Instituto Dante Pazzanese. Elsevier.

Agarwal, H. S. (2012). Standardized postoperative handover process improves outcomes in the intensive care unit: a model for operational sustainability and improved team performance. Crit. Care Med. 40(7); 2109-2115.

Almeida, S. L. (2017). Riscos da cirurgia cardíaca. Blog, Coronária, Fatores de Risco e Prevenção, Fatos e Mitos, Insuficiência Cardíaca. 2017.

Alves, T. E., Silva, M. G., Oliveira, L. C., Arrais, A. C \& Júnior, J. E. M. (2013). Atuação do Enfermeiro no Atendimento Emergencial aos Usuários Acometidos de Infarto Agudo do Miocárdio. Rev enferm UFPE on line., 7(1):176-83.

Assunçâo, M \& Oliveira, A. P. L. (2012) Manual de Normas e Rotinas de Procedimentos Endovasculares Extracardíacos. Serviço de Hemodinâmica do Hospital Getulio Vargas. Teresina-PI.

Barbosa, M. H., Moreira, T. M., Tavares, J. L., Andrade, E. V., Bitencourt, M. N, Freitas, K. B. C., et al. (20130. Complicações em pacientes submetido a Angioplastia Coronariana Transluminal Percutânea. Revista Eletronica Trimestal de Enfermagem. 2013.

Brunori, E. H. F. R., Lopes, C. T., Cavalcante, A. M. R. Z., Santos, V. B, Lopes, J. L \& Barros, A. L. B. L. (2014). Associação de Fatores de Risco Cardiovasculares com as Diferentes Apresentações da Síndrome Coronariana Aguda. Rev. Latino-Am. Enfermagem. 22(4):538-46.

Campos, L.A. (2011). Choque cardiogênico imediato de cirurgia cardíaca. Revista SOCERJ. 14(2); 115-11.

Cesário, J. M. S., Flauzino, V. H. P \& Mejia, J. V. C. (2020) Metodologia científica: Principais tipos de pesquisas e suas caraterísticas. Revista Científica Multidisciplinar Núcleo do Conhecimento. 11(5) 23-33.

Chaves, S.C. S., Brusamarello, T \& Huernermann, R. R. (2018). Health education within the hemodynamic service: an integrative review. Saúde Pesqui. 11(1):171-8.

Cheregatti, A. L \& Amorim, C. P. E. C. (2010). Enfermagem em Unidade de Terapia Intensiva. Martinari. 2010.

Costa, G. R., Cardoso, S. B., Sousa, L. L., Soares, T. R., Ferreira, A. K. A \& Lima, F. F. (2014). Practice nurses in service hemodynamic: an integrative review. R Interd. 7(3):157-64.

Costa, S. A., Cesário, J. M. S., Flauzino, V. H. P \& Mejia, J. V. C. (2021). Abordaje del dolor en pacientes con insuficiencia cardíaca. Evidentia. 18: e13219. http://ciberindex.com/c/ev/e13219

Costa, I. C., Cesário, J. M. S., Mejia, J. V. C \& Flauzino, V. H. P. (2021). Papel del equipo de enfermería durante la atención al paciente con sospecha de Infarto Agudo de miocardio. Evidentia, 18, e13197. http://ciberindex.com/c/ev/e13197

Cuoto, M. R. S. C., Cesário, J. M. S., Flauzino, V. H. P \& Mejia, J. V. C. (2020). El shock cardiogénico y sus implicaciones en el postoperatorio de la cirugía cardíaca. Ética de los cuidados. 13: e13005. http://ciberindex.com/index.php/et/article/view/e13005/e13005 
Dotto, J. I., Backes, D. S., Dalcin, C. B., Filho, W. D., Siqueira, H. C. H \& Zamberlan C. (2017). Systematization of nursing assistance: order, disorder or (re) organization?J Nurs UFPE on line. 11(10):3821-9.

Duarte, S. C. M., Stipp, M. A. C., Mesquita, M. G. R \& Silva, M. M. (2012). O cuidado de enfermagem no pós-operatório de cirurgia cardíaca: um estudo de caso. Esc. Anna Nery. 16 (4): 657-665.

Farias, F. B. B., Vidal, L. L., Farias, R. A. R \& Jesus, A. C. P. (2013). Cuidado humanizado em UTI: Desafios na visão dos profissionais de saúde. J. res.: fundam. care. 5(4):635-4.

Ferrari, R. F. R., Rodrigues, D. M. M. R., Baldissera, V. D. A., Pelloso, S. M \& Carreira, L. (2014). Applicability of the virginia henderson theory for foundations in nursing: weaknesses and potential. Arq Ciênc Saúde Unipar. 18(1):51-6.

Ferreira, E. B., Pereira, M. S., Souza, A. C. S., Almeida, C. C. O. F \& Taleb AC. (2016). Systematization of nursing care in the perspective of professional autonomy. Rev Rene. 17(1):86- 92.

Graeff, M. S., Goldmeier, S \& Pellanda LC. (2012). Síndrome Coronariana Aguda em Produtores de Tabaco: Fatores de Risco Prevalentes. Revista de Enfermagem da UFSM.

Hilário, T. S., Santos, S. M., Kruger, J., Goes, M. G., Casco, M. F \& Rabelo-Silva, E. R. (2017). Pain assessment and management in patients undergoing endovascular procedures in the catheterization laboratory. Rev Esc Enferm USP. 51:e03229.

Issa, A. F. C., Oliveira, G. M. M., Abreu, L. M., Rocha, R. M \& Esporcatte, R. (2015). Manual de Atualização e Conduta - Síndrome Coronariana Aguda (SCA). Editora PlanMark,

Kubrusly, L. F. (2010). Fatores de Risco em cirurgias cardíacas nos septuagenários. Portal do coração.

Lambert, P., Chaisson, K., Horton, S., Petrin, C., Marshall, E., Bowden, S., et al. (2017). Reducing Acute Kidney Injury Due to Contrast Material: How Nurses Can Improve Patient Safety. Crit Care Nurse. 37(1):13-26.

Lima, T. O. P., Vitorino, P. G. S., Mejia, J. V. C., Chã, N. V., Flauzino, V. H. P., Cusato, T. V., Gomes, D. M., Ribeiro, D. V., Hernandes, L. O., \& Cesário, J. M. S. (2021). Nursing care for chronic renal patients with heart disease on hemodialysis. Research, Society and Development, 10(10), e219101018100. https://doi.org/10.33448/rsd-v10i10.18100

Mansur, A. P \& Favarato, D. (2012). Mortalidade por Doenças Cardiovasculares no Brasil e na Região Metropolitana de São Paulo: Atualização. Arq. Brasileira de Cardiologia.

Mansur, A. P \& Favarato, D. (2016). Trends in Mortality Rate from Cardiovascular Disease in Brazil, 1980-2012. Arq Bras Cardiol. 10.5935/abc.20160077

Mosleh, S. M., Nidal, F. E \& Mona, M. A. A. (2017). Perceived learning needs according to patients who have undergone major coronary interventions and their nurses. J Clin Nurs. 2017 Feb; 26(3- 4):418-26.

Pereira, W. R., Flauzino, V. H. P., Mejia, J. V. C \& Cesário, J. M. S. (2021). Importancia de la atención de enfermería en el sector de hemodinámica: una revisión de la literatura brasileña. TESELA, Liderazgo y Gestión. (27): e13220. http://ciberindex.com/c/ts/e13220

Piegas, L.S \& Haddad N. (2010). Intervenção Coronariana Percutânea no Brasil. Resultados do Sistema Único de Saúde. Arquivos Brasileiros de Cardiologia, São Paulo, 2010.

Ponikowski, P., Voors, A. A., Anker, S. D., Bueno, H., Cleland, J. G. F., Coats AJS., et al. (2016). ESC Guidelines for the diagnosis and treatment of acute and chronic heart failure: The Task Force for the diagnosis and treatment of acute and chronic heart failure of the European Society of Cardiology (ESC)Developed with the special contribution of the Heart Failure Association (HFA) of the ESC. Eur Heart J. 2016 Jul 14;37(27):2129-2200.

Régis, A. P., Rosa, G. C. D., \& Lunelli,T. (2017). Cuidados de enfermagem no cateterismo cardíaco e angioplastia coronária: Desenvolvimento de um Instrumento. São Paulo: Revista Recien. 2017; 7(21):3-20.

Ribeiro, D. V., Vitorino, P. G. S., Mejia, J. V. C., Hernandes, L. de O., Lima, T. O. de P., Chã, N. V., Flauzino, V. H. de P., Cusato, T. V., Gomes, D. M., \& Cesário, J. M. S. (2021). Nursing conduct in pregnant women with heart disease contaminated by Covid-19. Research, Society and Development, 10(9), e29610918097. https://doi.org/10.33448/rsd-v10i9.18097

Rios, S. I. M., Flauzino, V. H. P., \& Cesário, J. M. S. (2021). A Importância Do Enfermeiro Na Assistência Ao Paciente No Cateterismo Cardíaco. Revista Científica Multidisciplinar Núcleo do Conhecimento. 2021; 5(3): 153-176. https://www.nucleodoconhecimento.com.br/saude/cateterismo-cardiaco, 10.32749/nucleodoconhecimento.com.br/saude/cateterismo-cardiaco

Santos, A. S. S \& Cesário, J. M. S. (2019). Atuação da enfermagem ao paciente com infarto agudo do miocárdio (IAM). São Paulo: Revista Recien. 9(27):6272 .

Santos, E. L., Dórea, S. N. A., Maciel, M. P. G. S., Santos, L. K. F., Silva, M. B \& Moraes, M. G. L. (2018). Assistência humanizada: percepção do enfermeiro intensivista. Rev baiana enferm. 32: e23680.

Shore-Lesserson, L., Baker, R. A., Ferraris, V. A., Greilich, P. E., Fitzgerald, D., Roman, P., et al. (2018). The Society of Thoracic Surgeons, The Society of Cardiovascular Anesthesiologists, and The American Society of Extracorporeal Technology: Clinical Practice Guidelines-Anticoagulation During Cardiopulmonary Bypass. Ann. Thorac. Surg. 105(2):650-62.

Silva, C., Sena, L., Rolim, I., Sousa, S \& Sardinha, A. (2017). Nursing care for patients with chronic health conditions: an integrative review. J res fudam care. 9(2):599-605. 
Research, Society and Development, v. 10, n. 12, e174101220220, 2021

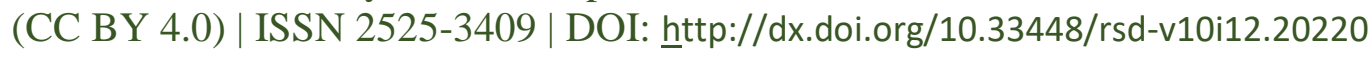

Silva, F. M., Pesaro, A. E., Franken, M \& Wajngarten, M. (2015). Tratamento Atual da Síndrome Coronariana Aguda sem Supradesnivelamento do Segmento ST. Einstein, São Paulo, einstein. 13(3):454-61.

Silva, G. S. P., Cesário, J. M. S., Flauzino, V. H. P \& Mejia, J. V. C. (2020). Conducta del equipo de enfermería en el sector hemodinámico ante los trámites administrativos y asistenciales. TESELA, Liderazgo y Gestión. (28): e13160.

Soares, C. B., Hoga, L. A. K., Peduzzi, M., Sangaleti, C., Yonekura, T \& Silva, D. R. A. D. (2014). Integrative Review: concepts and methods used in nursing. Rev Esc Enferm USP. 48(2):335-45.

Soares, G. M. T., Ferreira, D. C. S., Gonçalves, M. P. C., Alves, T. G. S., David, F. L., Henriques, K. M. C., et al. (2011). Prevalência das Principais Complicações Pós-Operatórias em Cirurgias Cardíacas. Revista Brasileira de Cardiologia. 24(3); 139-146.

Sousa, S. E. S., Flauzino, V. H. P., Cesário, J. M. S. (2020). A Importância Da Humanização Da Equipe De Enfermagem Na Unidade De Terapia Intensiva. Revista Científica Multidisciplinar Núcleo do Conhecimento. 2020; 5(3): 196-211. https://www.nucleodoconhecimento.com.br/saude/humanizacao-da-equipe, 10.32749/nucleodoconhecimento.com.br/saude/humanizacao-da-equipe

Szelkowski, L. A., Puri, N. K., Singh, R., \& Massimiano, P. S. (2015). Current trends in preoperative, intraoperative, and postoperative care of the adult cardiac surgery patient. Current Problems in Surgery. 2015. 5(2) 531-569. 